Activated Corrosion Product Analysis. Analytical Approach 
This report was prepared as an account of work sponsored by an agency of the United States Government. Neither the United States Government nor any agency thereof, nor any of their employees, makes any warranty, express or implied, or assumes any legal liability or responsibility for the accuracy, completeness, or usefulness of any information, apparatus, product, or process disclosed, or represents that its use would not infringe privately owned rights. Reference herein to any specific commercial product, process, or service by trade name, trademark, manufacturer, or otherwise, does not necessarily constitute or imply its endorsement, recommendation, or favoring by the United States Government or any agency thereof. The views and opinions of authors expressed herein do not necessarily state or reflect those of the United States Government or any agency thereof. 


\title{
ACTIVATED CORROSION PRODUCT ANALYSIS. ANALYTICAL APPROACH
}

\author{
Stanislav Golubov, UT, MSTD \\ Jeremy Busby, MSTD \\ Roger Stoller, MSTD \\ U.S. ITER Technical Project Officer \\ Andrei Petrov
}

Date Published: January 2010

Prepared by

OAK RIDGE NATIONAL LABORATORY

Oak Ridge, Tennessee 37831-6283

managed by

UT-BATTELLE, LLC

for the

U.S. DEPARTMENT OF ENERGY

under contract DE-AC05-00OR22725 



\section{CONTENTS}

Page

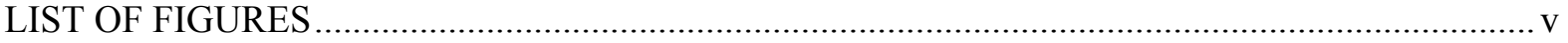

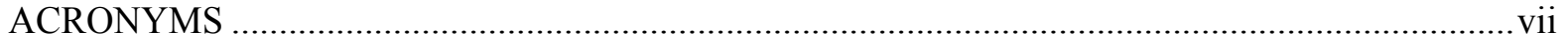

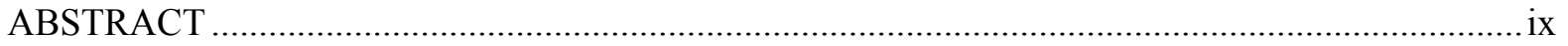

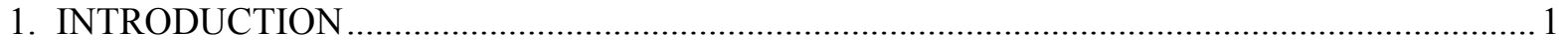

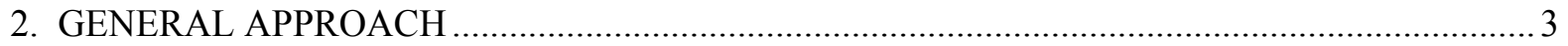

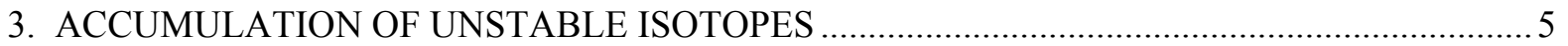

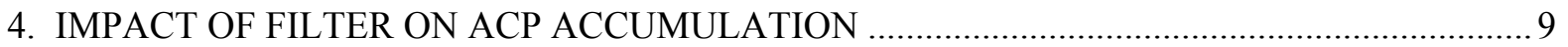

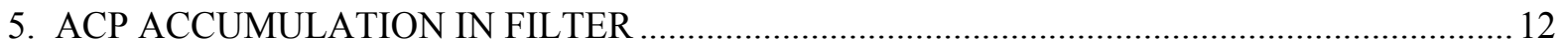

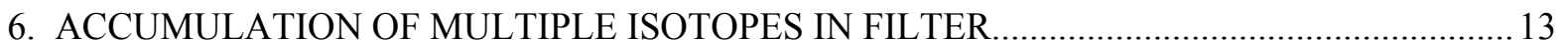

7. ACCUMULATION OF ISOTOPES WITH TIME DEPENDENT ACTIVITY SOURCE ............. 14

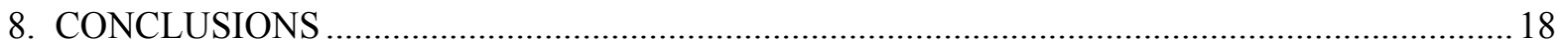

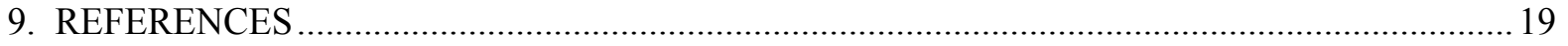





\section{LIST OF FIGURES}

Figure

Page

1 ACP distribution in water along the pipe calculated for $\tau / T=1$ and for 1,3 , and 5 cycles 7

ACP distribution in water along the pipe calculated for $\tau / T=10$ and for the number of cycles ranging from 1 to 50

Total ACP accumulation in water calculated by using Eq. (18) as a function of the ratio $\tau / T$ for different number of cycles

$4 \quad$ Relationship between the filter efficiency $\gamma$ and the efficiency parameter $\alpha$

5 ACP distribution in water along a pipe calculated for $\tau / T=10$ and number of cycles ranging from 1 to 50

6 Total ACP accumulation as a function of the ratio $\tau / T$ for different number of cycles and filter efficiency $\gamma=1 \%$

Total ACP accumulated in the filter as a function of the ratio $\tau / T$ for a different number of cycles and filter efficiency $\gamma=1 \%$ 



\section{ACRONYMS}

ACP Activated corrosion products

ALARA As Low As Reasonably Achievable

CEA French Atomic Energy Commission

ITER International Thermonuclear Experimental Reactor

PWR Pressurized Water Reactor

TCWS Tokamak Cooling Water System 



\begin{abstract}
The presence of activated corrosion products (ACPs) in a water cooling system is a key factor in the licensing of ITER and affects nuclear classification, which governs design and operation. The objective of this study is to develop a method to accurately estimate radionuclide concentrations during ITER operation in support of nuclear classification. A brief overview of the PACTITER numerical code, which is currently used for ACP estimation, is presented. An alternative analytical approach for calculation of ACPs, which can also be used for validation of existing numerical codes, including PACTITER, has been proposed. A continuity equation describing the kinetics of accumulation of radioactive isotopes in a water cooling system in the form of a closed ring has been formulated, taking into account the following processes: production of radioactive elements and their decay, filtration, and ACP accumulation in filter system. Additional work is needed to more accurately assess the ACP inventory in the cooling water system, including more accurate simulation of the Tokamak cooling water system (TCWS) operating cycle and consideration of material corrosion, release, and deposition rates.
\end{abstract}





\section{INTRODUCTION}

Activated corrosion products (ACP) will be present in ITER in-vessel and vacuum vessel coolant loops as well as in coolant loops related to test modules and auxiliary heating or diagnostics equipment. All surfaces that come in contact with water in the ITER Tokamak cooling water system (TCWS) will corrode and release material to the cooling water. For components exposed to the neutron flux (copper and stainless steel in particular), these corrosion products will be radioactive and could contribute to occupational exposure, routine release effluents to the environment, and potential releases during accidents. This fact has made the ACP inventory evaluation a critical task for ensuring ITER public and occupational safety.

ACP levels are a key factor in the licensing of ITER and may have a large impact on its design and operation in order to keep ITER in the lowest possible safety category. As a result, accurate estimates of radioactivity concentrations for various components have to be established beforehand. This information can also help guide the operation of ITER to ensure that activity inventories are below regulatory limits and that As Low As Reasonably Achievable (ALARA) principles are met. This may be accomplished by estimating the ACP inventory accumulated in filters and resins of TCWS cleanup system (Chemical and Volume Control System).

The PACTITER code, which exists as several versions, is the main instrument used for calculations and is derived from the PACTOLE code, developed by the French Atomic Energy Commission (CEA) for predicting ACPs in pressurized water reactor (PWR) primary circuits [1,2]. The operating conditions, material compositions, and water chemistry of the various Primary Heat Transfer Systems of ITER required that the PACTOLE code be modified. PACTITER was developed based on dedicated experiments, namely, those devoted to determining copper solubility and stainless steel release under ITER primary cooling systems conditions, which are rather different from those in a PWR (i.e., water chemistry and temperatures). The PACTITER code has been used extensively in support of the ITER Generic Site Safety Report in the field of accident analysis and worker collective dose assessment.

The PACTITER code is based on a control volume approach; the primary circuit is represented by an arrangement of several volumes in which transient mass balance equations are solved:

$$
\frac{d m_{i}}{d t}+\left(\dot{m}_{s}-\dot{m}_{e}\right)=\sum_{\text {Source }} J_{m}-\sum_{\text {Sink }} J_{m},
$$


where $m_{i}$ is the mass of the $i$ th isotope in the form of corrosion products in a given volume, $t$ is the time, $\dot{m}_{s}-\dot{m}_{e}$ is the convective term (balance between input and output), and $J_{m}$ is the exchange mass rate between two different volume elements. The convective term does not account for nuclear reactions (e.g., N-16, 17, and C-14 production) which involve mass production and loss within the considered volume. Although such an approach provides a feasible way for carrying out the calculations, it does not provide a simple way for checking the accuracy of the calculations. In the framework of such a model, it is not possible to obtain simple analytical solutions which can be used as benchmarks for the more detailed numerical calculations. Thus, an alternate approach for the activity calculations which may provide such an opportunity could be very useful [3].

An initial analysis was performed during FY 2007 to quantify the level of activated corrosion products being released to the ITER cooling water system [4]. The EASY-2003 code was used to simulate the activation of 316 stainless steel and copper as a function of ITER operating time and neutron fluence. The key long-lived isotopes generated in stainless steel included Co-58, Tc-99, Co-60, Co-57, and Mn-54, consistent with previous studies. The simulations for copper predicted that $\mathrm{Cu}-64, \mathrm{Co}-60, \mathrm{Ni}-63, \mathrm{Zn}-65$, Co-58, and Fe-59 will be the dominant, long-lived isotopes, which is also consistent with previous work. The simulations show that the activity level produced from copper will remain constant throughout the ITER lifetime as production and decay rates reach a steady state almost immediately. For stainless steel, however, activity will build throughout the projected lifetime.

These activation rates were then used to calculate the release rate of activated material into the cooling water system by simply multiplying the activation rate by the corrosion release rates calculated in other studies [5,6]. An additional study has been performed to assess the decay of ACPs as the water flows through the system [7]. This analysis indicates that the total ACP inventory in the water cooling system is lower than originally calculated, but is still a significant quantity.

While the initial simulations and analysis provide useful information, the current effort is still very simplistic relative to the complexity of the actual ITER design. Additional work is needed to more accurately assess the ACP inventory in the cooling water system, including more accurate simulation of TCWS operating cycle and consideration of ACP deposition. 
The objective of this work is development of a simplified model for calculating ACPs which permits analytical treatment of the problem and analysis of these processes. Results of this analysis will be compared with PACTITER results to ensure consistency.

\section{GENERAL APPROACH}

In order to formulate the problem in a rigorous way, let us assume that a vacuum vessel coolant loop can be illustrated as a pipe in the form of a ring, with a radius $R$ and length of $L=2 \pi R$. Let us also assume that the activation area on this pipe may be considered as a point activity source located at the beginning of a coordinate system, $x=0$, where $x$ is a coordinate measured along the cooling ring (note that the points with the coordinates of $x=0$ and $x=2 \pi R$ are equal). Let us label the velocity of water flow, the period of water cycle, total number of " $i$ " isotope atoms produced per unit time, and half-life time of the isotope as $V, T, Q_{i}^{0}$ and $\tau_{i}$, respectively. Note that the values $L, V$ and $T$ are connected to each other by the following equation:

$$
L=V T \text {. }
$$

Let us also introduce an activity unit, $Q_{i}=Q_{i}^{0} \mathrm{~T}$, which is equal to the total amount of " $i$ " isotopes added to water for a period of $T$, that is, during one cycle. The parameter $Q_{i}^{0}$ (and $Q_{i}$ ) is a function of several parameters, including isotope production and decay/removal, as well as analytically and/or experimentally determined material corrosion, release, and deposition rates. ${ }^{*}$ The ratio $Q_{i} / \mathrm{L}$ describes the activity density, that is, an average amount of " $i$ " isotope atoms injected into water of a unit length of the pipe during one cycle with a unit of measurement of "atoms per meter."

To formulate the main equation describing accumulation of corrosion elements in water flowing in the pipe, let us consider first the simplest case when there is one non-radioactive element only $(\tau=\infty)$. Let us assume also that the velocity of the isotope is equal to that of cooling water; that is, it moves along the pipe with the velocity $V$. Introducing the density of the isotope activity (i.e., the total amount of isotope atoms) at a point with coordinate $x$ at time $t, f(x, t)$ (index " $i$ " is omitted for simplicity), an equation describing distribution of the isotope along the ring is given by

$$
\frac{d f(x, t)}{\partial t}=-V \frac{d f(x, t)}{\partial x} .
$$

The general solution of Eq. (2) has a form

${ }^{*}$ Consideration of corrosion, release, and deposition rates of material is beyond the scope of this report. 


$$
f(x, t)=\psi(x-V t),
$$

where $\psi(z)$ is an arbitrary function, showing that a certain "state" (isotope activity in this case) moves in a space with velocity $V$. The form of function $\psi(z)$ in Eq. (3) depends on initial and boundary conditions. Taking into account that the pipe has the form of a ring, the boundary condition in the case is

$$
f(x, t)=f(x+L, t) .
$$

Initial conditions depend on the problem under consideration. In the simplest case, when an isotope is initially homogenously distributed along the ring with concentration $C_{0}$, the initial condition is given by

$$
f(x)_{t=0}=C_{0} .
$$

The solution of Eq. (2) in this case is

$$
f(x, t)=C_{0},
$$

which is physically correct since movement of stable isotopes along the ring cannot change their distribution.

The situation with activation of the cooling pipe under ITER conditions is more complicated since radioactive isotopes are produced continuously in a certain part of the pipe. For the sake of simplicity, let us assume that the size of the isotope "production area" is much smaller than that of the pipe size. In this case a reasonable approximation can be obtained assuming that the activity source can be considered to be dimensionless at a certain coordinate on the pipe, $x=x_{s}$. If, for example, the isotope source is located at the beginning of coordinate, $x_{s}=0$, an equation describing the process of activity accumulation can be readily obtained from Eq. (2) by adding the source term

$$
\frac{d f(x, t)}{\partial t}=\frac{Q}{L} \delta(x)-V \frac{d f(x, t)}{\partial x},
$$

where $\delta(x)$ is a Dirac delta function. The initial condition for the density function $f_{i}(x, t)$ in the case is given by

$$
f(x, t)_{t=0}=0 .
$$

Equation (7) with the initial and boundary conditions given by Eqs. (8) and (4) can be solved. Thus for a time less than the period, $t<T$, taking into account Eq. (8), the density $f(x, t)$ is given by 


$$
f(x, t)_{\mid 0 \leq t<T}=\frac{Q}{L} \theta(V t-x) \equiv\left\{\begin{array}{ll}
\frac{Q}{L}, & x=V t<L \\
0, & V t<x \leq L
\end{array},\right.
$$

where the function $\theta(z)$ is the Heaviside step function $(\theta(z)=1$ at $z \geq 0$ and $\theta(z)=0$ at $z<0)$. At the

time equal to the period, $t=T$, the isotope distribution is equal to $f(x, T)=\frac{Q}{L}$. In the same way one can find that at $t=n T$, where $n$ is an arbitrary integer, the density is equal to $f(x, n T)=n \frac{Q}{L}$. The total amount of the element accumulated during this time can be easily calculated by integrating along the ring:

$$
R_{n}=\int_{0}^{L} f(x, n T) d x=n Q .
$$

Equation (10) describes the conservation law: the total amount of isotope in this case is proportional to the number of cycles, taking into account that the value of $Q$ is equal to the amount of the element produced during the period.

The analysis just presented demonstrates how a problem of impurity accumulation in water circulating in pipe with can be rigorously formulated. Moreover, it shows how Eq. (2) for the density $f(x, t)$ can be generalized to describe different mechanisms affecting isotope accumulation. In the next section, this approach will be applied to the ACP problem, taking into account radioactive decay and water filtration.

\section{ACCUMULATION OF UNSTABLE ISOTOPES}

Taking into account the half-life, $\tau$, an equation describing the accumulation of an isotope can be obtained by adding a decay term $\frac{f(x, t)}{\tau}$ in Eq. (7). Thus it takes the following form:

$$
\frac{d f(x, t)}{\partial t}=\frac{Q}{L} \delta(x)-V \frac{d f(x, t)}{\partial x}-\frac{f(x, t)}{\tau} .
$$

The general solution of Eq. (11) is given by

$$
f(x, t)=\Psi(x-V t) e^{-t / \tau},
$$


where $\Psi(z)$ is an arbitrary function. Taking into account the initial and boundary conditions formulated in Sect. 2, the density $f(x, t)$ at $t<T$ takes the following form:

$$
f(x, t)_{\mid 0 \leq t<T}=\frac{Q}{L} e^{-\frac{x}{V \tau}} \theta(V t-x) \equiv\left\{\begin{array}{ll}
\frac{Q}{L} e^{-\frac{x}{V \tau}}, & x=V t<L \\
0, & V t<x \leq L
\end{array} .\right.
$$

From Eq. (13) it follows that ACP accumulated during the first period (i.e., when $t=T$ ) is equal to

$$
f_{1}(x)=f(x, T)=\frac{Q}{L} e^{-\frac{x}{V \tau}} .
$$

The total ACP accumulated in the ring for the first period can be readily calculated:

$$
R_{1}=\int_{0}^{L} f_{1}(x) d x=Q\left(1-e^{-T / \tau}\right)
$$

Note that in the limiting case of the stable isotope $\left(\tau \rightarrow \infty\right.$ ), the activity $R_{1} \rightarrow Q$, as it has to be in accordance with the conservation law [see Eq. (10)]. Since the period $T$ is orders of magnitude less than the operation time of ITER, it can be considered as a natural time unit for the calculations presented below.

It can be easily shown that ACP accumulation during the second and third periods (i.e., at $t=2 T$ and $t=3 T$, is given by

$$
\begin{aligned}
& f_{2}(x)=\frac{Q}{L}\left(1+e^{-T / \tau}\right) e^{-\frac{x}{V \tau}} \\
& f_{3}(x)=\frac{Q}{L}\left(1+e^{-T / \tau}+e^{-2 T / \tau}\right) e^{-\frac{x}{V \tau}}
\end{aligned}
$$

Equation (16) allows us to generalize the solution for an arbitrary number of periods, $t=n T$,

$$
f_{n}(x)=\frac{Q}{L}\left(1+e^{-T / \tau}+e^{-2 T / \tau}+\ldots+e^{-(n-1) T / \tau}\right) e^{-\frac{x}{V \tau}} \equiv \frac{Q}{L} \frac{\left(1-e^{-n T / \tau}\right)}{\left(1-e^{-T / \tau}\right)} e^{-\frac{x}{V \tau}} .
$$

Total ACP accumulated after $n$ cycles can be calculated as follows:

$$
R_{n}=\int_{0}^{L} f_{n}(x) d x=Q \frac{\tau}{T} \frac{1-e^{-n T / \tau}}{1-e^{-T / \tau}}\left(1-e^{-T / \tau}\right) \equiv Q \frac{\tau}{T}\left(1-e^{-n T / \tau}\right) .
$$

Equations (17) and (18) are the closed form solutions for the isotope activity distribution along the pipe and the total amount accumulated in the whole pipe, respectively. 
In the two limiting cases of (1) a very short half-life $(\tau<<n T)$ and (2) a very long one $(\tau>>n T)$, Eq. (18) leads to the following results:

$$
R_{n} \approx\left\{\begin{array}{ll}
Q \frac{\tau}{T}, & \frac{\tau}{n T}<<1 \\
Q n, & \frac{\tau}{n T}>>1
\end{array} .\right.
$$

In the first case, the activity reaches a steady-state value which is proportional to the half-life, whereas in the second case it increases proportional to the number of cycles (i.e., irradiation time). In both cases the results show that the analysis presented above gives a reasonable description of the activity accumulation.

Results for the ACP distribution along the pipe calculated using Eq. (17) are presented in Figs. 1 and 2 for two cases, $\tau / T=1$ and 10 , respectively, and for a different number of cycles.

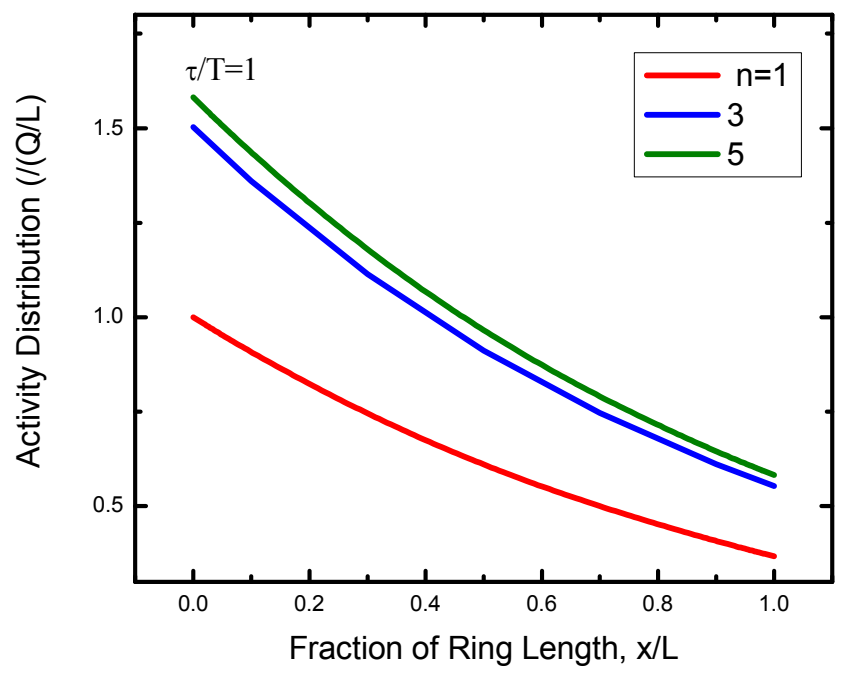

Fig. 1. ACP distribution in water along the pipe calculated for $\tau / T=1$ and for 1,3 , and 5 cycles. The case $n=5$ represents the saturation distribution in water, which does not change for $n>5$. 


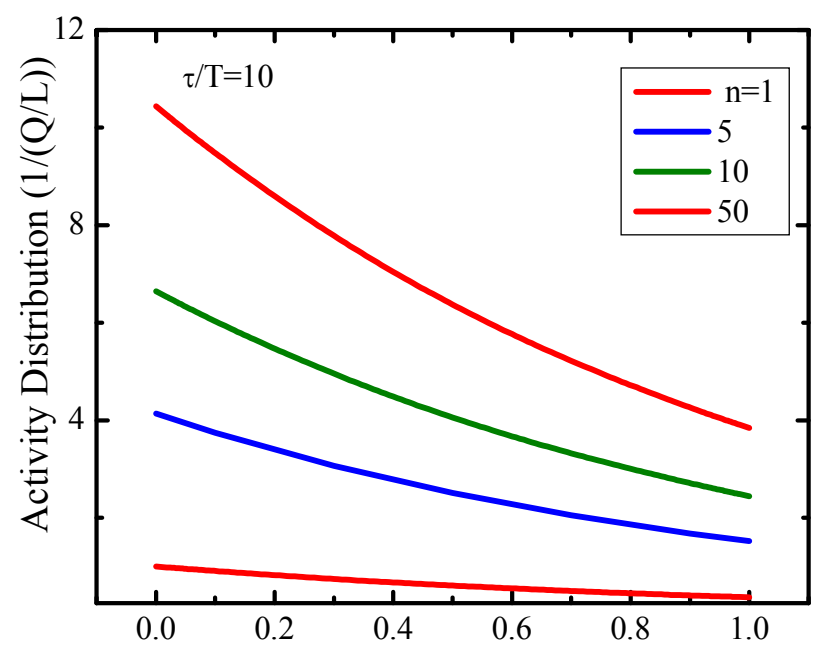

Fraction of Ring Length, $\mathrm{x} / \mathrm{L}$

Fig. 2. ACP distribution in water along the pipe calculated for $\tau / T=10$ and for the number of cycles ranging from 1 to 50 . The case $n=50$ represents the saturation distribution in water, which does not change for $n>50$.

For the general case in which the half-life has an arbitrary magnitude, the total amount of the ACP accumulated in the system depends on the number of cycles, that is, the operation time. In Fig. 3, the function $R_{n}$ is plotted as a function of the ratio $\tau / T$ when the number of cycles ranges from 1 to 1000 . As can be seen from the plot, the activity tends to saturate with an increasing number of cycles at a level that depends on the half-life.

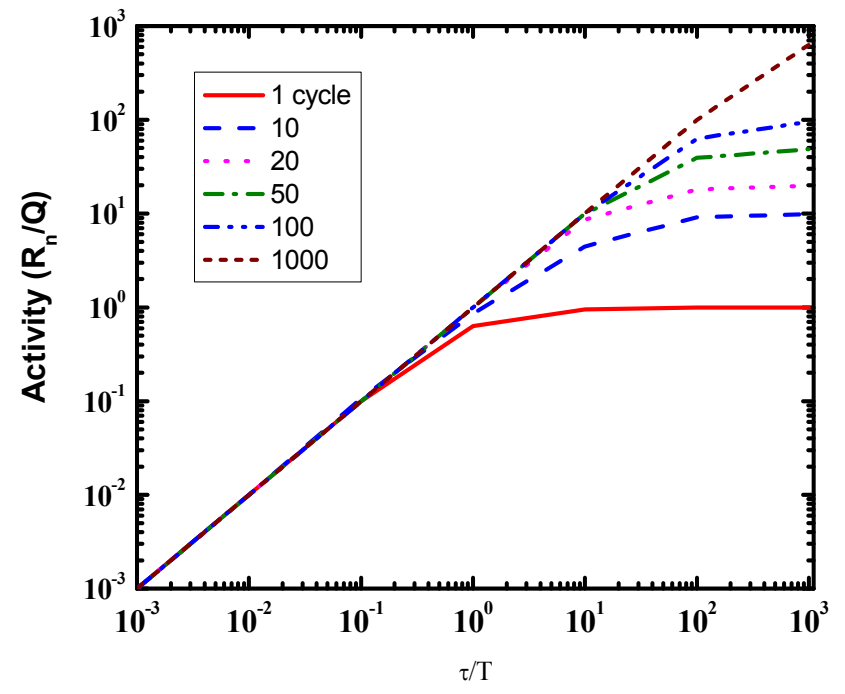

Fig. 3. Total ACP accumulation in water calculated using Eq. (18) as a function of the ratio $\tau / T$ for a different number of cycles. 


\section{IMPACT OF FILTER ON ACP ACCUMULATION}

Let us assume that the size of a filter is small compared to the loop size; thus, it can be described as a dimensionless point located at a certain position on the water pipe. Defining a filter with efficiency $\alpha$, that is located at $x=x_{0}$, one finds that an equation describing ACP accumulation in the ring can be obtained by generalizing Eq. (11) in the following way:

$$
\frac{d f(x, t)}{\partial t}=\frac{Q}{L} \delta(x)-V \frac{d f(x, t)}{\partial x}-\frac{f(x, t)}{\tau}+V f(x, t)\left(1-\frac{1}{\alpha}\right) \delta\left(x-x_{0}\right) .
$$

The last term on the right-hand side of Eq. (20) accounts for a decrease in the activity in $\alpha$ time after passing the filter. Note that the magnitude of the filter efficiency defined in this way is $\alpha \geq 1$. The filter efficiency measured in percent, as normally used in practice, say $\gamma$, is related to $\alpha$ in the following way:

$$
\gamma=\frac{\alpha-1}{\alpha} 100 \%
$$

The relationship between these two parameters is presented in Fig. 4, showing that a very small deviation of the parameter $\alpha$ from unity results in a large increase in the filter efficiency $\gamma$.

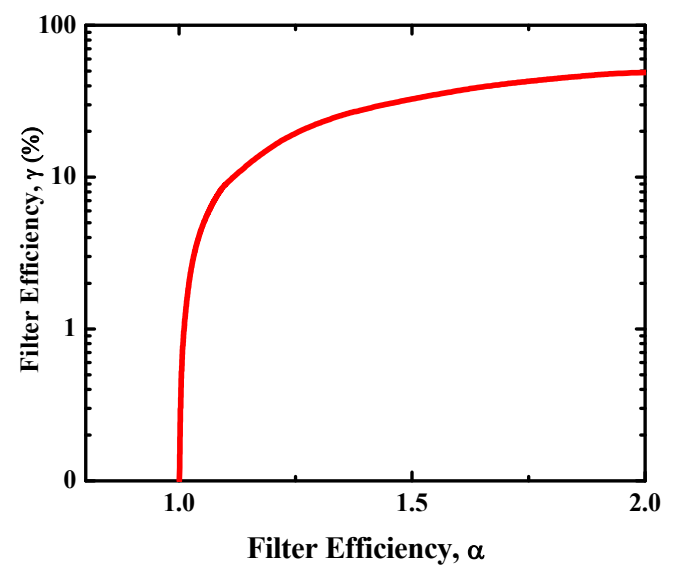

Fig. 4. Relationship between the filter efficiency $\gamma$ and the efficiency parameter $\alpha$.

Solution of Eq. (21) can be obtained the same way as it has been done above. It can be shown that after the first period, $t=T$, the ACP density, $F(x, t)$ (the function $F(x, t)$ is used to distinguish it from the case considered in previous paragraphs), is given by 


$$
F(x, T)=\frac{Q}{L}\left\{\begin{array}{ll}
e^{-\frac{x}{V \tau}}, & x \leq x_{0} \\
\frac{1}{\alpha} e^{-\frac{x}{V \tau}}, & x>x_{0}
\end{array} .\right.
$$

After the second and third periods, $t=2 T$ and $t=3$, the distributions $F(x, t)$ are given by

$$
\begin{gathered}
F_{2}(x)=\frac{Q}{L}\left(1+\frac{1}{\alpha} e^{-\frac{T}{\tau}}\right)\left\{\begin{array}{lc}
e^{-\frac{x}{V \tau}}, & x \leq x_{0} \\
\frac{1}{\alpha} e^{-\frac{x}{V \tau}}, & x>x_{0}
\end{array}\right. \\
F_{3}(x)=\frac{Q}{L}\left[1+\left(1+\frac{1}{\alpha} e^{-\frac{T}{\tau}}\right) \frac{1}{\alpha} e^{-\frac{T}{\tau}}\right]\left\{\begin{array}{lc}
e^{-\frac{x}{V \tau}}, & x \leq x_{0} \\
\frac{1}{\alpha} e^{-\frac{x}{V \tau}}, & x>x_{0}
\end{array} \equiv\right. \\
\frac{Q}{L}\left[1+\frac{e^{-\frac{T}{\tau}}}{\alpha}+\left(\frac{e^{-\frac{T}{\tau}}}{\alpha}\right)^{2}\right]\left\{\begin{array}{lc}
e^{-\frac{x}{V \tau}}, & x \leq x_{0} \\
\frac{1}{\alpha} e^{-\frac{x}{V \tau}}, & x>x_{0}
\end{array}\right.
\end{gathered}
$$

Thus after the $n$ cycles, $t=n T$, one can find that

$$
\begin{aligned}
& F_{n}(x)=\frac{Q}{L}\left(1+\left(\frac{e^{-\frac{T}{\tau}}}{\alpha}\right)+\left(\frac{e^{-\frac{T}{\tau}}}{\alpha}\right)^{2}+\ldots+\left(\frac{e^{-\frac{T}{\tau}}}{\alpha}\right)^{n-1}\right)\left\{\begin{array}{ll}
e^{-\frac{x}{V \tau}}, & x \leq x_{0} \\
\frac{1}{\alpha} e^{-\frac{x}{V \tau}}, & x>x_{0}
\end{array} \equiv\right. \\
& \frac{Q}{L} \frac{1-\left(\frac{e^{-T / \tau}}{\alpha}\right)^{n}}{1-\left(\frac{e^{-T / \tau}}{\alpha}\right)}\left\{\begin{array}{ll}
e^{-\frac{x}{V \tau}}, & x \leq x_{0} \\
\frac{1}{\alpha} e^{-\frac{x}{V \tau}}, & x>x_{0}
\end{array} .\right.
\end{aligned}
$$

Using Eq. (25) one can find the total ACP accumulated after $n$ cycles is given by

$$
R_{n}=Q \frac{\tau}{T} \frac{1-\left(\frac{e^{-T / \tau}}{\alpha}\right)^{n}}{1-\left(\frac{e^{-T / \tau}}{\alpha}\right)}\left[\left(1-e^{-T_{0} / \tau}\right)+\frac{1}{\alpha}\left(e^{-T_{0} / \tau}-e^{-T / \tau}\right)\right]
$$


where $T_{0}=x_{0} / V$. Equations (25) and (26) are closed form solutions for the activity accumulation in the case under consideration. Note that in the limiting case of $\alpha=1$ (no filter), Eqs. (25) and (26) are transformed to Eqs. (17) and (18).

The ACP distribution along the ring for the case of $\gamma \approx 10 \%$ is illustrated in Fig. 5. The same parameters were employed as used to calculate $f(x, t)$ for the case when the filter is absent (Fig. 1b).

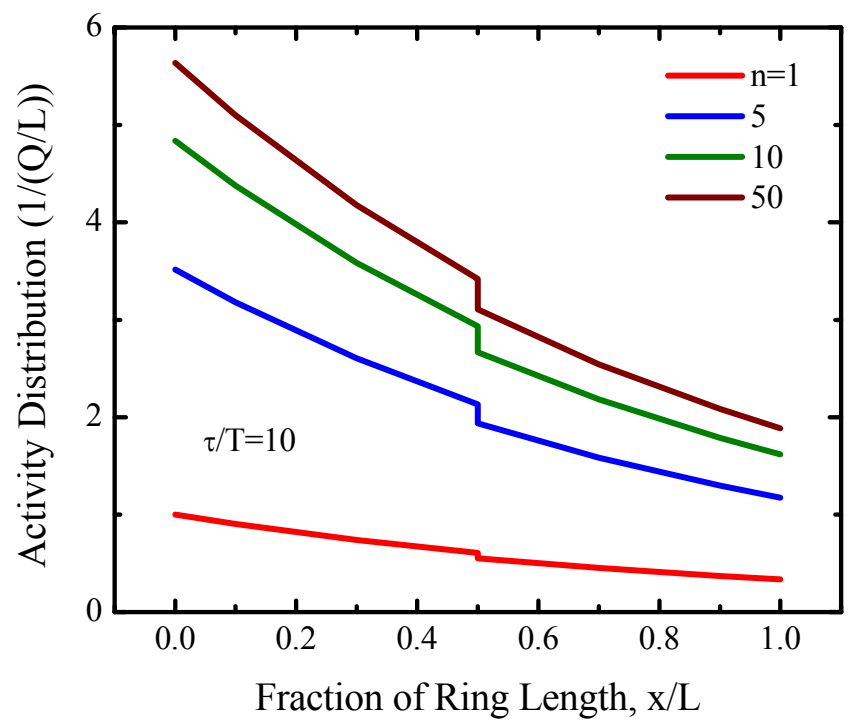

Fig. 5. ACP distribution in water along a pipe calculated for $\tau / T=10$ and the number of cycles ranging from 1 to 50. The case $n=50$ represents a steady-state distribution, which does not change for $n>50$.

The total ACP accumulated in the ring after a different number of cycles as calculated using Eq. (26) is presented in Fig. 6. For comparison, the total activity accumulated after the same number of cycles calculated using Eq. (18) (no filter case) is also presented in Fig. 6. It can be seen from the plot that the filter significantly decreases activity in the case of the long-lived isotope $(\tau / T>>1)$. Moreover, after a certain number of cycles the amount of activity is practically saturated. Correspondingly, the activity accumulated in the filter increases with increasing operation time. 


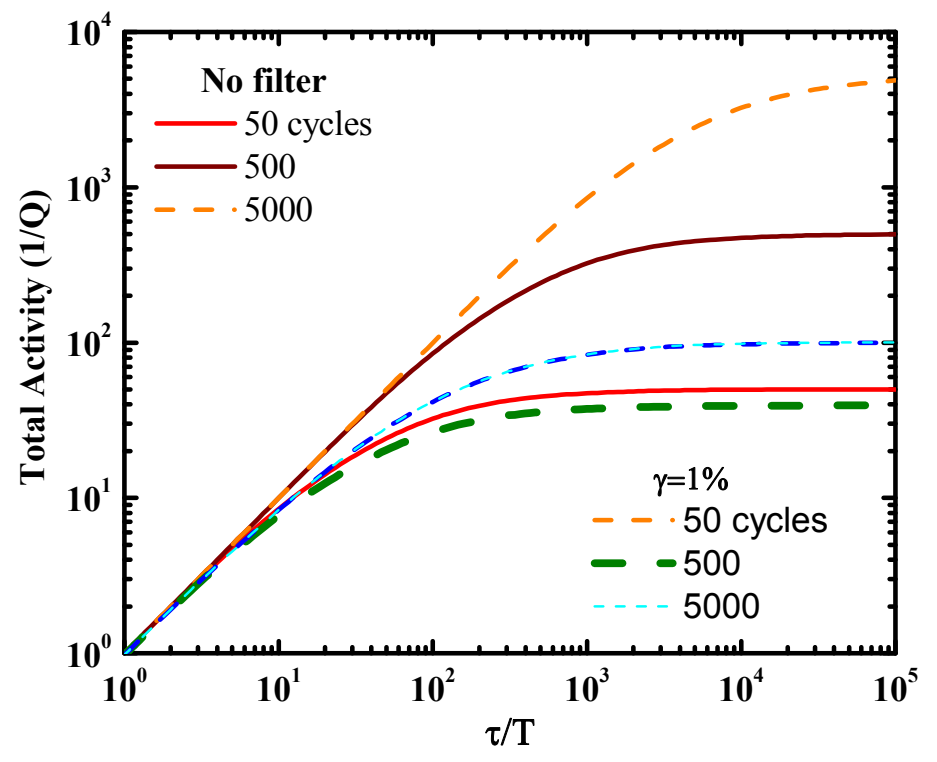

Fig. 6. Total ACP accumulation as a function of the ratio $\tau / T$ for a different number of cycles and filter efficiency $\gamma=\mathbf{1 \%}$. The calculations have been done for the case when $x_{0}=L / 2\left(T_{0}=T / 2\right)$.

\section{ACP ACCUMULATION IN FILTER}

The ability to calculate total ACP accumulation in the filter, after $n$ cycles, $\Phi_{n}$, is very important for practical purposes. It can be calculated by integrating the difference between the activity distributions described by Eqs. (17) (no filter case) and (25) (filter case). One can find that

$$
\begin{aligned}
& \Phi_{n}(t=n T)=\frac{Q \tau}{T}\left\{\left[\frac{\left(1-e^{-n T / \tau}\right)}{\left(1-e^{-T / \tau}\right)}-\frac{1-\left(\frac{e^{-T / \tau}}{\alpha}\right)^{n}}{1-\left(\frac{e^{-T / \tau}}{\alpha}\right)}\right]\left(1-e^{-T_{0} / \tau}\right)+\right. \\
& \left.\left[\frac{\left(1-e^{-n T / \tau}\right)}{\left(1-e^{-T / \tau}\right)}-\frac{1}{\alpha} \frac{1-\left(\frac{e^{-T / \tau}}{\alpha}\right)^{n}}{1-\left(\frac{e^{-T / \tau}}{\alpha}\right)}\right]\left(e^{-T_{0} / \tau}-e^{-T / \tau}\right)\right\} .
\end{aligned}
$$

An example of the ACP calculated accumulation in a filter with an efficiency of $1 \%$ is presented in Fig. 7 for a number of cycles ranging from 50 to 5000 . 


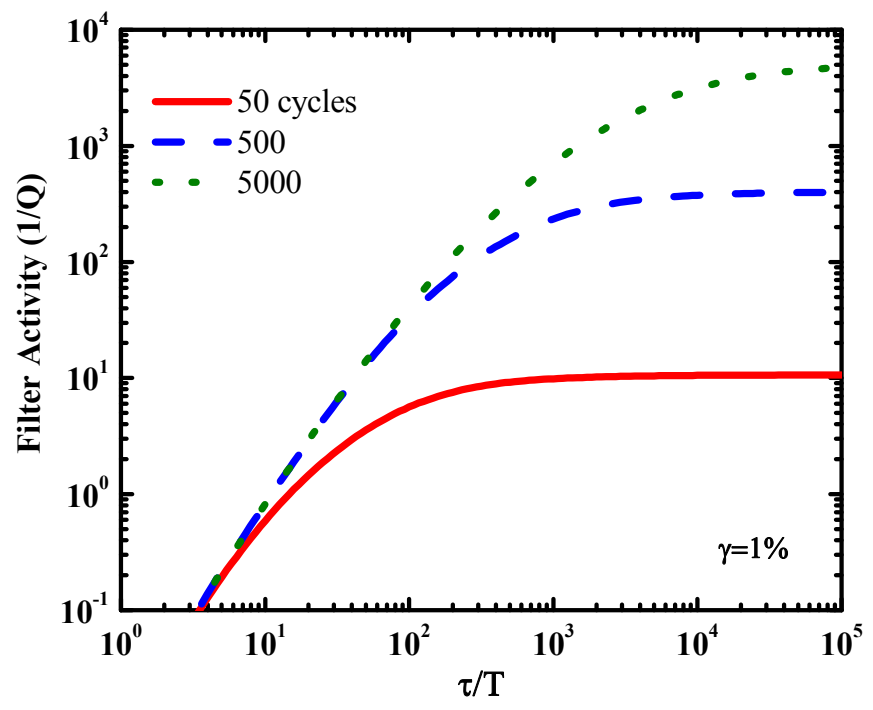

Fig. 7. Total ACP accumulated in the filter as a function of the ratio $\tau / T$ for a different number of cycles and filter efficiency $\gamma=1 \%$.

\section{ACCUMULATION OF MULTIPLE ISOTOPES IN FILTER}

Assuming that production and accumulation of different isotopes are independent, the activity distribution along the cooling pipe, $F_{n}^{\text {tot }}(x, t)$, and total activity after $n$ cycles, $R_{n}^{\text {tot }}$, can be calculated as a linear superposition of activities of particular isotopes:

$$
F_{n}^{t o t}(x)=\sum_{i=1}^{m} F_{n}^{i}(x), \quad R_{n}^{t o t}=\sum_{i=1}^{m} R_{n}^{i},
$$

where $m$ is the total number of the isotopes. Thus the activity distribution in the pipe after $n$ cycles, $F_{n}^{t o t}(x, t)$, takes the following form:

$$
F_{n}^{t o t}(x)=\frac{1}{L} \sum_{i=1}^{I} Q_{i}\left\{\frac{1-\left(\frac{e^{-T / \tau_{i}}}{\alpha_{i}}\right)^{n}}{1-\left(\frac{e^{-T / \tau_{i}}}{\alpha_{i}}\right)}\left[\begin{array}{cc}
e^{-\frac{x}{V \tau_{i}}}, & x \leq x_{0} \\
\frac{1}{\alpha} e^{-\frac{x}{V \tau_{i}}}, & x>x_{0}
\end{array}\right]\right\}
$$

where $Q_{i} \tau_{i}$ and $\alpha_{i}$ are the rates of isotope production, their half-life times, and the filter efficiency. Equation (29) is given for the case when the filter location is the same for all isotopes. The total activity for $n$ cycles in this case can be calculated as follows: 


$$
R_{n}^{t o t}=\int_{0}^{L} F_{n}^{t o t}(x) d x=\frac{1}{T} \sum_{i=1}^{I} \tau_{i} Q_{i}\left\{\frac{1-\left(\frac{e^{-T / \tau_{i}}}{\alpha_{i}}\right)^{n}}{1-\left(\frac{e^{-T / \tau_{i}}}{\alpha_{i}}\right)}\left[\left(1-e^{-T_{0} / \tau_{i}}\right)+\frac{1}{\alpha_{i}}\left(e^{-T_{0} / \tau_{i}}-e^{-T / \tau_{i}}\right)\right]\right\}
$$

The total activity accumulated in the filter during $n$ cycles is given by

$$
\begin{aligned}
& \Phi_{n}=\sum_{i=1}^{I} Q_{i} \frac{\tau_{i}}{T}\left\{\left[\frac{\left(1-e^{-n T / \tau_{i}}\right)}{\left(1-e^{-T / \tau_{i}}\right)}-\frac{1-\left(\frac{e^{-T / \tau_{i}}}{\alpha}\right)^{n}}{1-\left(\frac{e^{-T / \tau_{i}}}{\alpha}\right)}\right]\left(1-e^{-\frac{x_{0}}{L}\left(T / \tau_{i}\right)}\right)+\right. \\
& \left.\left[\frac{\left(1-e^{-n T / \tau_{i}}\right)}{\left(1-e^{-T / \tau_{i}}\right)}-\frac{1}{\alpha} \frac{1-\left(\frac{e^{-T / \tau_{i}}}{\alpha_{i}}\right)^{n}}{1-\left(\frac{e^{-T / \tau_{i}}}{\alpha_{i}}\right)}\right]\left(e^{-\frac{x_{0}}{L}\left(T / \tau_{i}\right)}-e^{-T / \tau_{i}}\right)\right\} .
\end{aligned}
$$

Note that Eqs. (29)-(31) describe the activity accumulation in the cooling ring filter in the case when a source of activity and a filter can be considered as the point objects. This is a good approximation as long as the length of pipe exposed to irradiation is much less than the total length of pipe. If this is not the case, the equation may be generalized to take this into account.

\section{ACCUMULATION OF ISOTOPES WITH TIME-DEPENDENT ACTIVITY SOURCE}

More generally, three parameters may depend on time: (1) the rate of production of an " $i$ " isotope, $Q_{i}(t)$; (2) the velocity of cooling water, $V(t)$; and (3) the filter efficiency, $\alpha_{i}(t)$. The solution is more complicated in this case since practically all coefficients in the main equation for activity distribution may depend on time:

$$
\frac{d f(x, t)}{\partial t}=\frac{Q(t)}{L} \delta(x)-V(t) \frac{d f(x, t)}{\partial x}-\frac{f(x, t)}{\tau}+V(t) f(x, t)\left(1-\frac{1}{\alpha(t)}\right) \delta\left(x-x_{0}\right) .
$$

ACP accumulation under such conditions is of very practical interest. First, it permits calculating the activity accumulation for different scenarios of ITER operation, which may be useful in choosing the final operational design. Second, it will provide more a realistic description of activity accumulation during the actual operation of ITER. This case will be considered here, and it may be shown that 
Eq. (32) can be also solved analytically. For the sake of simplicity, only the time dependence of the rate of ACP production is taken into account and presented in this report.

The main equation in this case takes the following form:

$$
\frac{d f(x, t)}{\partial t}=\frac{Q(t)}{L} \delta(x)-V \frac{d f(x, t)}{\partial x}-\frac{f(x, t)}{\tau} V f(x, t)\left(1-\frac{1}{\alpha}\right) \delta\left(x-x_{0}\right) .
$$

Like the previous analysis, we assume that the period of water circulation, $T=L / V$, is much smaller than that of the operation time; thus, it can be employed as a natural time step.

After one cycle (i.e., when $t=T$ ), the ACP is distributed as

$$
F_{1}(x)=\frac{e^{-\frac{x}{V \tau}}}{L} Q\left(T-\frac{x}{V}\right)\left\{\begin{array}{ll}
1, & x \leq x_{0} \\
\frac{1}{\alpha}, & x>x_{0}
\end{array} .\right.
$$

The total ACP accumulated in the pipe can be obtained by integrating Eq. (34) to yield

$$
R_{1}=\frac{1}{L}\left\{\int_{0}^{x_{0}} Q\left(T-\frac{x}{V}\right) e^{-\frac{x}{V \tau}} d x+\frac{1}{\alpha} \int_{x 0}^{L} Q\left(T-\frac{x}{V}\right) e^{-\frac{x}{V \tau}} d x\right\} .
$$

After two cycles $(t=2 T)$, the ACP distribution takes the following form:

$$
F_{2}(x)=\frac{e^{-\frac{x}{V \tau}}}{L}\left\{\begin{array}{lc}
Q\left(2 T-\frac{x}{V}\right)+\frac{e^{-T / \tau}}{\alpha} Q\left(T-\frac{x}{V}\right), & 0<x \leq x_{0} \\
\frac{1}{\alpha}\left[Q\left(2 T-\frac{x}{V}\right)+\frac{e^{-T / \tau}}{\alpha} Q\left(T-\frac{x}{V}\right)\right], & x_{0}<x \leq L
\end{array} .\right.
$$

The ACP accumulation during the two periods is given by

$$
\begin{aligned}
R_{2}= & \frac{1}{L} \int_{0}^{x_{0}}\left[Q\left(2 T-\frac{x}{V}\right)+\frac{e^{-T / \tau}}{\alpha} \int_{x 0}^{L} Q\left(2 T-\frac{x}{V}\right)\right] e^{-\frac{x}{V \tau}} d x+ \\
& \frac{1}{\alpha L} \int_{x_{0}}^{L}\left[Q\left(T-\frac{x}{V}\right) e^{-\frac{x}{V \tau}}+\frac{e^{-T / \tau}}{\alpha} Q\left(T-\frac{x}{V}\right) e^{-\frac{x}{V \tau}}\right] d x
\end{aligned}
$$

For the three periods, the ACP distribution is given by

$$
F_{3}(x, 3 T)=\frac{e^{-\frac{x}{V \tau}}}{L}\left\{\begin{array}{l}
Q\left(3 T-\frac{x}{V}\right)+\frac{e^{-T / \tau}}{\alpha} Q\left(2 T-\frac{x}{V}\right)+\frac{e^{-2 T / \tau}}{\alpha^{2}} Q\left(T-\frac{x}{V}\right), \quad 0<x \leq x_{0} \\
\frac{1}{\alpha}\left[Q\left(3 T-\frac{x}{V}\right)+\frac{e^{-T / \tau}}{\alpha} Q\left(2 T-\frac{x}{V}\right)+\frac{e^{-2 T / \tau}}{\alpha^{2}} Q\left(T-\frac{x}{V}\right)\right], \quad x_{0}<x \leq L
\end{array} .\right.
$$


The structure of Eqs. (34), (36), and (38) permits us to write the ACP distribution function and total ACP accumulation after $n$ cycles as follows:

$$
\begin{gathered}
F_{n}(x)=\frac{e^{-\frac{x}{V \tau}}}{L}\left\{\begin{array}{l}
\sum_{k=1}^{n} \frac{e^{-(n-k) T / \tau}}{\alpha^{(n-k)}} Q\left(k T-\frac{x}{V}\right), \quad 0<x \leq x_{0} \\
\frac{1}{\alpha} \sum_{k=1}^{n} \frac{e^{-(n-k) T / \tau}}{\alpha^{(n-k)}} Q\left(k T-\frac{x}{V}\right), \quad x_{0}<x \leq L
\end{array}\right. \\
R_{n}=\int_{0}^{L} F_{n}(x, n T) d x=\frac{1}{L}\left\{\sum_{k=1}^{n} \frac{e^{-(n-k) T / \tau}}{\alpha^{(n-k)}}\left[\int_{0}^{x_{0}} Q\left(k T-\frac{x}{V}\right) e^{-\frac{x}{V \tau}} d x+\frac{1}{\alpha} \int_{x_{0}}^{L} Q\left(k T-\frac{x}{V}\right) e^{-\frac{x}{V \tau}} d x\right]\right\} .
\end{gathered}
$$

Equations (39) and (40) are the closed form solutions for the ACP distribution in a cooling pipe and the total ACP accumulated in the pipe at any time for the case when the rate of activity production is an arbitrary function of time. ACP accumulation in the filter, $\Phi_{n}$, can be calculated using Eq. (40). As was shown above, $\Phi_{n}$ is equal to the difference between the activity accumulated in the cases with and without a filter. Equations for the last case can be readily obtained from Eqs. (39) and (40), taking $\alpha=1$. Equations (39) and (40) in that case take the following form:

$$
\begin{gathered}
F_{n}(x)_{\mid \alpha=1}=\frac{e^{-\frac{x}{V \tau}}}{L} \sum_{k=1}^{n} e^{-(n-k) T / \tau} Q\left(k T-\frac{x}{V}\right), \quad 0<x \leq L, \\
R_{n \mid(\alpha=1)}=\int_{0}^{L} F_{n}(x)_{\mid \alpha=1} d x=\frac{1}{L}\left\{\sum_{k=1}^{n} e^{-(n-k) T / \tau} \int_{0}^{L} Q\left(k T-\frac{x}{V}\right) e^{-\frac{x}{V \tau}} d x\right\} .
\end{gathered}
$$

Thus, one can find that the total ACP accumulation in the filter is given by

$$
\begin{aligned}
& \Phi_{n}=R_{n \mid \alpha=1}-R_{n}=\frac{1}{L} \sum_{k=1}^{n} e^{-(n-k) T / \tau}\left(1-\frac{1}{\alpha^{(n-k)}}\right) \int_{0}^{x_{0}} Q\left(k T-\frac{x}{V}\right) e^{-\frac{x}{V \tau}} d x+ \\
& \frac{1}{L} \sum_{k=1}^{n} e^{-(n-k) T / \tau}\left(1-\frac{1}{\alpha^{(n-k+1)}}\right) \int_{x_{0}}^{L} Q\left(k T-\frac{x}{V}\right) e^{-\frac{x}{V \tau}} d x .
\end{aligned}
$$

Note that in the limiting case when the source of activity is independent of time, $Q(t)=Q$, Eqs. (39) and (40) transform to

$$
F_{n}(x)=\frac{Q e^{-\frac{x}{V \tau}}}{L} \begin{cases}\sum_{k=1}^{n} \frac{e^{-(n-k) T / \tau}}{\alpha^{(n-k)}}, & 0<x \leq x_{0} \\ \sum_{k=1}^{n} \frac{e^{-(n-k) T / \tau}}{\alpha^{(n-k+1)}}, & x_{0}<x \leq L\end{cases}
$$




$$
R_{n}=\frac{Q \tau}{T} \frac{1-\left(\frac{e^{-\tau / T}}{\alpha}\right)^{n}}{1-\left(\frac{e^{-\tau / T}}{\alpha}\right)}\left\{\left(1-e^{-\frac{x_{0}}{L}\left(\frac{T}{\tau}\right)}\right)+\frac{1}{\alpha}\left(e^{-\frac{x_{0}}{L}\left(\frac{T}{\tau}\right)}-e^{-\frac{T}{\tau}}\right)\right\},
$$

which are identical to the Eqs. (25) and (26).

It has to be emphasized that the model considered above is rather simplified since all essential elements of the cooling system, that is, the activity source and filter, are considered to be dimensionless. However, the main equation formulated, Eq. (32), can be generalized for more realistic conditions. For example, the ACP source and filter terms can be presented as follows:

$$
\begin{aligned}
& \frac{1}{L} \sum_{j=1}^{J} Q_{j}(t) \delta\left(x-x_{j}\right) \\
& V(t) f(x, t) \sum_{k=1}^{K}\left(1-\frac{1}{\alpha(t)_{k}}\right) \delta\left(x-x_{k}\right),
\end{aligned}
$$

where $Q_{j}(t), x_{j}$ are the rates of ACP production and locations of these sources and $\alpha(t)_{k}, x_{k}$ are a filter efficiency and their location. In this case the source of ACP production can be described in a way which is more realistic and to some extent similar to that used in the PACTITER code. Equation (32) can be also generalized for the case when precipitation of ACP has to be taken into account. In this case an additional equation for evolution of the precipitates has to be added to the generalized continuity equation in accordance with the physics of the process.

It should be noted that due to the complexity of these analytical solutions, it may be more practical to solve the continuity equation using numerical integration. Indeed, Eq. (32) can be presented in the following form:

$$
\Delta f(x, t)=\Delta t\left\{\frac{Q(t)}{L} \delta(x)-V(t) \frac{d f(x, t)}{\partial x}-\frac{f(x, t)}{\tau}+V(t) f(x, t)\left(1-\frac{1}{\alpha(t)}\right) \delta\left(x-x_{0}\right)\right\},
$$

which is suitable for numerical solution by any of the different methods used to integrate differential equations of this type and can be applied to calculate accumulation of isotopes in water given timedependent activity source. 


\section{CONCLUSIONS}

Accurate estimation of the concentration of radioactivity in different components of TCWS loops will be the key factor in licensing of ITER for construction and operation. An analytical approach for calculation of ACP accumulation has been proposed. A continuity equation describing the kinetics of accumulation of radioactive isotopes in a water cooling system in the form of a closed ring has been formulated, taking into account the following processes: production of radioactive elements and their decay and filtration. The main advantage of the equation formulated is that it provides the ability to solve the equation in a closed form. It has been shown that analytical solutions can be obtained even in the case when an operational parameter such as the rate of ACP production is an arbitrary function of the operation time. Additional work is needed to more accurately assess the ACP inventory in the cooling water system, including more accurate simulation of the TCWS operating cycle and development of experimental data or analytical methods for determining material corrosion, release, and deposition rates. This approach can be used to cross-check the results of numerical calculations obtained by using different approaches, and to design an alternative code to PACTITER for analytical calculation of ACP accumulation in the cooling water system. 


\section{REFERENCES}

1. L. Di Pace et al., Development of the PACTITER Code and its Application to Safety Analysis of ITER Primary Cooling Water System, Fusion Engineering and Design, Vol. 82, 2007, p. 237.

2. L. Di Pace, Overview of PACTITER Code, Meeting on ACP Assessment for ITER, October 08, 2008, Oak Ridge, TN, USA.

3. S. Golubov et al., "Modeling of Activated Corrosion Product Transport: A Theoretical Approach," paper presented at the AIChE 2008 Annual Meeting, November 16-21, 2008, Philadelphia, PA, USA.

4. J. Busby et al., Selection of Construction Materials, Type of Piping, and ACP Assessment for ITER TCWS, US ITER 12100-TD0002-R00, ORNL, Oak Ridge, TN, USA, 2007.

5. A. Molander, Corrosion and Water Chemistry Aspects Concerning the Tokamak Cooling Water Systems of ITER, STUDSVIK/N-06/186, Studsvik Nuclear AB, Sweden, 2006.

6. K. Lueven, Copper Alloy Corrosion in High-Temperature Water, Task T511, Subtask 3, ITER, Cadarache, France, 2001.

7. O. Jonas and L. Machemer, Corrosion, Activation, water Chemistry, and Materials-ITER Tokamak Cooling Water Systems - Interim Assessment, US ITER, ORNL, Oak Ridge, TN, USA, 2008. 



\section{INTERNAL DISTRIBUTION}

1. J. B. Berry

2. J. T. Busby

3. S. I. Golubov

4. R. K. Nanstad

5. Yu. N. Osetsky
6. A.Y. Petrov

7. R. E. Stoller

8. S. J. Zinkle

9. U.S. ITER TCWS

10. File-USIPO DCC-RC

\section{EXTERNAL DISTRIBUTION}

12. L. Di Pace, ENEA, C.R. Frascati, via Enrico Fermi 45, Frascati (Rome), I-00044, Italy

13. V. Barabash, ITER Organization, Building 519, Cadarache, 13108 St Paul Les Durance Cedex, France

14. W. Curd, ITER Organization, Building 525B, Cadarache, 13108 St Paul Les Durance Cedex, France

15. G. Dell'Orco, ITER Organization, Building 525B, Cadarache, 13108 St Paul Les Durance Cedex, France 